\title{
Adaptation of ASTC in a Correlated Rayleigh Frequency-Selective Fading Channels in OFDM systems with PAPR Reduction
}

\author{
Ahmed BANNOUR*, Mohamed Lassaad AMMARI ${ }^{\dagger}$ and Ridha BOUALLEGUE ${ }^{\ddagger}$ \\ * $\ddagger$ SUP’COM, 6’Tel Unit, Higher School of Communication of Tunis, Tunis, Tunisia \\ $\dagger$ SUPCOM, 6Tel Laboratory, National Engineering School of Sousse, Sousse, Tunisia \\ Ahmed.Bannour@isima.rnu. tn, mohamed. ammari@eniso.rnu. tn, ridha. \\ bouallegue@supcom.rnu.tn
}

\begin{abstract}
In this paper we suggest to use the ASTC (Algebraic Space Time Codes) as powerful coding technique for IEEE 802.11x OFDM standard combined with PAPR reduction scheme. ASTC with their very Algebraic- construction based on Quaternionic algebra, have a full rate, full diversity, non-vanishing constant minimum determinant for increasing spectral efficiency, uniform average transmitted energy per antenna and good shaping, readily lend themselves to high data rate situations. However, by their construction they require a nonselective flat fading channels belonging to narrow-band systems. In fact, such systems are not readily found in airs interfaces. In open air we have what are commonly called frequency-selective fading channels. As a matter of fact, it becomes extremely necessary to harness their power for wide-band systems.
\end{abstract}

\section{Index Terms}

ASTC codes, Analytic Bit Error Rate, Doppler frequency, OFDM, Peak to Average Reduction, MIMO

\section{INTRODUCTION}

Fourth generation $(4 \mathrm{G})$ wireless technology is expected to provide high bit-rate multimedia communication capability. Nevertheless, high data rate applications require data transmission over broadband frequency selective channels, which cause severe Inter-Symbol Interference (ISI). Orthogonal frequency division multiplexing (OFDM) is well accepted as an effective technique to mitigate ISI with absence of complex equalization [1]-[3]. OFDM system performance can be widely improved with channel coding. OFDM has many advantages, which make it an attractive scheme for high-speed transmission links. However, one major difficulty is OFDM's large Peak to Average Power Ratio (PAPR). Those are created by the coherent summation of the OFDM subcarriers. When $N$ signals are added with the same phase, they produce a peak power that is $N$ times the average power. These large peaks cause saturation in the power amplifiers, leading to intermodulation products among the subcarrier and disturbing out of band energy. Hence, it becomes worth while reducing PAPR. Towards this end there are several proposals such as clipping, coding and peak windowing [4]. Respectively, reduction of PAPR comes at a price of performance degradation, mainly in terms of rate and BER [5]. This paper proposes to use the ASTC codes as powerful coding techniques for IEEE 802.11x OFDM standard combined with PAPR scheme. ASTC codes can for out a good solution first to overcome the disadvantage of OFDM modulations and second to keep a robustness regarding the BER performances. Theses codes were proposed in 2004 by [6]-[8], a $2 \times 2$ STBC obtained using a division algebra. ASTC could exceeds the Alamouti codes performances, thanks to it's algebraic construction which guarantees three major advantages : First, a nonzero lower bound on the coding gain, which is independent of the spectral efficiency (non-vanishing determinant).

10.5121/ijwmn.2010.2209 
Second, the shaping constraint, with an objective to make sure that the codes are energy efficient. Third, uniform average transmitted energy per antenna is also required.

The outline of this paper is organized as follows. In section II, we describe the system and channel models. Section III, deals with a PAPR reduction scheme. Then, in section IV we analyze the empirical BER for an ASTC-MIMO-OFDM system. Last but not least, section V covers, simulation analysis for different scenarios and compares them to one another. Finally, a sum up is given in section VI.

\section{AStC Codes in a Frequency-Selective Channel Context}

We consider a coherent system over a frequency-selective correlated Rayleigh fading MIMO channel $2 \times$ 2. The overall schematic diagram of ASTC-MIMO-OFDM transceiver is depicted in Fig. 1. The transmitted

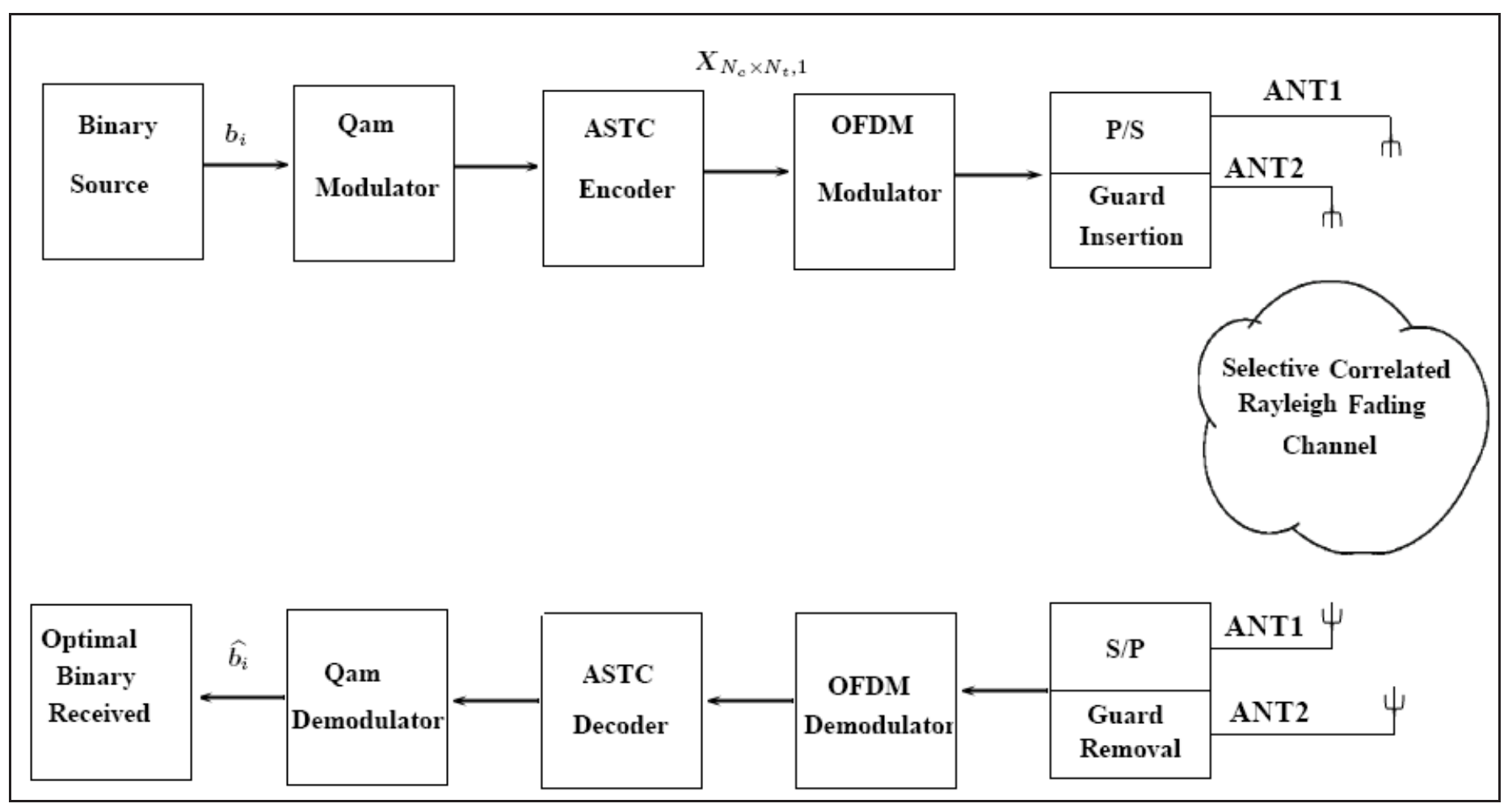

Figure 1. Block diagram of Joint-Coded ASTC-OFDM system.

binary source sequence $b_{i}$ of length $L$ is modulated using the QAM-4 modulator. Each information sequence at time $n_{i}$.

$$
\boldsymbol{v}_{n_{i}, n_{i+1}}=\left[s_{\left(2 n_{i+1}\right)-3}, s_{\left(2 n_{i+1}\right)-2}, s_{\left(2 n_{i+1}\right)-1}, s_{\left(2 n_{i+1}\right)}\right]^{T}
$$

is encoded by the ASTC encoder into two stream constellations represented by the code word $\boldsymbol{X}_{N_{c} \times N_{t}}$ where $N_{t}$ refers to number of transmitted antennas and $N_{c}$ is the number of used subcarriers. By their construction the channel was under the Quasi-Static assumption, and does not take into account neither the time variation nor the selectivity channel case. To spread their power regarding the bit rate and the BER performance into the selective channel case with time variation, we introduce the best perfect algebraic code known as Golden codes with other tow well famous algebraic space time codes, TAST and DAST as in [7].

\section{A. Golden Encoder}

The code was proposed in 2004 by [7], [9] a $2 \times 2$ STBC obtained using a division algebra, which is full rate, full diversity, and has a nonzero lower bound on its coding gain, which does not depend on the 
constellation size. The code word is written as :

$$
\boldsymbol{X}_{n_{i}}=\frac{1}{\sqrt{5}}\left(\begin{array}{cc}
\alpha\left(\boldsymbol{v}_{n_{i}}(1)+\theta \boldsymbol{v}_{n_{i}}(2)\right) & \alpha\left(\boldsymbol{v}_{n_{i}}(3)+\theta \boldsymbol{v}_{n_{i}}(4)\right) \\
\bar{\alpha}\left(\boldsymbol{v}_{n_{i}}(3)+\bar{\theta} \boldsymbol{v}_{n_{i}}(4)\right) & \bar{\alpha}\left(\boldsymbol{v}_{n_{i}}(1)+\bar{\theta} \boldsymbol{v}_{n_{i}}(2)\right)
\end{array}\right)
$$

where

$$
\theta=\frac{1+\sqrt{5}}{2} \quad \bar{\theta}=\frac{1-\sqrt{5}}{2} \alpha=1+i-i \theta \quad \bar{\alpha}=1+i-i \bar{\theta}
$$

\section{B. TAST Encoder}

As shown in [7] [9], the TAST code is a $2 \times 2$ space time algebraic code obtained using the integer algebra, with rate $R=N_{t}=2$ Symbole/uc(usedcodeword), and diversity $D=N_{t} \times N_{r}=4$. Each space time layer is associated with his proper algebraic space $\varphi$ in order to alleviate the problem of ISI (Inter-Symbol-Interferences). The code word is expressed as :

$$
\boldsymbol{X}_{\text {Tast }}=\frac{1}{\sqrt{2}}\left(\begin{array}{cc}
(\boldsymbol{v}(1)+\theta \boldsymbol{v}(2)) & \varphi(\boldsymbol{v}(3)+\theta \boldsymbol{v}(4)) \\
\varphi(\boldsymbol{v}(3)-\theta \boldsymbol{v}(4)) & (\boldsymbol{v}(1)-\theta \boldsymbol{v}(2))
\end{array}\right)
$$

where

$$
\theta=\exp (i \lambda) \quad \lambda \in \Re \quad \varphi=\theta^{2}
$$

\section{DAST Encoder}

The DAST code is a $2 \times 2$ diagonal space time algebraic code obtained using the turned constellations of integer algebra, with rate 1 Symbole/uc, and full diversity. The code word is described as follows [9]:

$$
\boldsymbol{X}_{\text {Dast }}=\boldsymbol{H}_{n_{t}} \cdot \operatorname{diag}\left(\boldsymbol{M} \boldsymbol{v}_{n_{t}}\right)
$$

where

$$
\boldsymbol{M}=\frac{1}{\sqrt{2}}\left(\begin{array}{cc}
1 & \theta \\
1 & -\theta
\end{array}\right) \quad \theta=\exp (i \pi / 4) \quad \boldsymbol{H}_{n_{t}}=\text { Hadamard matrix of } n_{t} \text { degree }
$$

$M$ is the rotation matrix of $n_{t}=2$ degree.

\section{Time variation Representation of ASTC}

As mentioned above the ASTC works under the non time variant channel assumption, which is not commonly the case. In fact, we can re-express the total code word $\boldsymbol{X}_{(\mathbf{G o l d e n}, \text { Tast,Dast) }}$ to a vector $\boldsymbol{x}_{n_{i}}^{p}$ of length $\boldsymbol{l}=4$ with $p=\in$ (Golden, Tast, Dast) at time $\left(n_{i}, n_{i+1}\right)$ as the follows:

$$
\begin{gathered}
\boldsymbol{x}_{n_{i}}^{p}=\frac{1}{\sqrt{5}}\left(\begin{array}{c}
(\alpha(\boldsymbol{v}(1)+\theta \boldsymbol{v}(2)))_{\left(n_{i}, 1\right)} \\
(\bar{\alpha}(\boldsymbol{v}(3)+\bar{\theta} \boldsymbol{v}(4)))_{\left(n_{i+1}, 1\right)} \\
(\alpha(\boldsymbol{v}(3)+\theta \boldsymbol{v}(4)))_{\left(n_{i}, 2\right)} \\
(\bar{\alpha}(\boldsymbol{v}(1)+\bar{\theta} \boldsymbol{v}(2)))_{\left(n_{i+1}, 2\right)}
\end{array}\right) \quad \boldsymbol{x}_{n_{i}}^{p}=\frac{1}{\sqrt{2}}\left(\begin{array}{c}
((\boldsymbol{v}(1)+\theta \boldsymbol{v}(2)))_{\left(n_{i}, 1\right)} \\
(\varphi(\boldsymbol{v}(3)-\theta \boldsymbol{v}(4)))_{\left(n_{i+1}, 1\right)} \\
(\alpha(\boldsymbol{v}(3)+\theta \boldsymbol{v}(4)))_{\left(n_{i}, 2\right)} \\
(\bar{\alpha}(\boldsymbol{v}(1)+\bar{\theta} \boldsymbol{v}(2)))_{\left(n_{i+1}, 2\right)}
\end{array}\right) \\
\boldsymbol{x}_{n_{i}}^{p}=\frac{1}{\sqrt{2}}\left(\begin{array}{c}
((\boldsymbol{v}(1)+\theta \boldsymbol{v}(2)))_{\left(n_{i}, 1\right)} \\
((\boldsymbol{v}(1)+\theta \boldsymbol{v}(2)))_{\left(n_{i+1}, 1\right)} \\
((-\boldsymbol{v}(1)+\theta \boldsymbol{v}(2)))_{\left(n_{i}, 2\right)} \\
((\boldsymbol{v}(1)-\theta \boldsymbol{v}(2)))_{\left(n_{i+1}, 2\right)}
\end{array}\right)
\end{gathered}
$$

That means at each $\left(n_{i}, j\right)$ we transmit a part of the code word on the $j^{\text {th }}$ antennas. 


\section{E. Frequency-Selective Correlated Rayleigh Fading Channels}

Wide-Band systems are commonly a Frequency-Selective Correlated Rayleigh Fading Channels. However the ASTC requires a nonselective flat fading channels belonging to narrow-band systems. To alleviate this problem let focus on lattice representation of a Frequency-Selective Correlated Rayleigh Fading Channels. We adopt here the Clarke channel model [10]. The received signal is the sum of $q$ waves, we take into account the Doppler shifts effect. To obtain a correlated Rayleigh fading channel, the autocorrelation function of $\left\{h_{k}^{j}\right\}$ process is given by:

$$
\begin{aligned}
r_{h} & =E\left[h_{k}^{j} h_{k-q}^{j}\right] \\
& =\sigma_{h}^{2} \exp \left(j 2 \pi f_{c} q\right) J_{0}\left(2 \pi f_{m} q T_{s}\right)
\end{aligned}
$$

where $J_{0}$ is the Bessel function with zero order, $f_{m}$ is the maximum Doppler shift and $j$ is the antenna's number. If we guess that we have $N_{t}\left(N_{c}+N_{g}\right)$ subcarrier used and the channel length is $L \leq N_{t}\left(N_{c}+N_{g}\right)$ we can represent the channel in function of the correlated Rayleigh taps $h_{k}$ which verify (6), where $N_{g}$ refers to the number of guard subcarrier and $N_{t}$ to the number of transmitted antennas as follows:

$$
\boldsymbol{H}=\left(\begin{array}{cccccccc}
h_{0} & 0 & \ldots & \ldots & \ldots & \ldots & \ldots & 0 \\
h_{1} & \ddots & & & \vdots & & & \vdots \\
h_{2} & & \ddots & \ldots & 0 & \ldots & & \vdots \\
\vdots & & & \ddots & \vdots & & & \vdots \\
h_{L-1} & \ldots & \ldots & \ldots & h_{0} & & & \vdots \\
0 & \ddots & & & & \ddots & & \vdots \\
\vdots & & \ddots & & & & \ddots & 0 \\
0 & \ldots & \ldots & h_{L-1} & \ldots & \ldots & \ldots & h_{0}
\end{array}\right)
$$

In order to use the ASTC codes properly, we need to convert the channel $\boldsymbol{H}$ into $N_{t} \times\left(N_{c}+N_{g}\right)$ non selective sub-channels, The core idea is that the wide-band frequency selective MIMO channel by means of the MIMO-OFDM processing is transferred to a number of parallel flat fading MIMO channels. In fact each code word $\boldsymbol{x}_{n_{i}}^{p}$ will be modulated within the $N_{c} N_{t}$ sub-channels, without loss of generality, now we are assuming that all subcarriers are used :

$$
\boldsymbol{z}=\sqrt{N_{c}}\left(\boldsymbol{F}^{-1} \otimes \boldsymbol{I}_{N_{t}}\right) \boldsymbol{x}_{N c N_{t}, 1}^{p}
$$

This transforms the frequency domain vector $\boldsymbol{x}_{N_{c} N_{t}, 1}$ into the time domain. where $\otimes$ represents the Kronecker product and $\boldsymbol{F}^{-1}$ represent the IFFT Matrix defined as :

$$
\boldsymbol{F}^{-1}=\frac{1}{N_{c}}\left(\begin{array}{ccccc}
1 & 1 & 1 & 1 & 1 \\
1 & w^{-1} & w^{-1} & \ldots & w^{-\left(N_{c}-1\right)} \\
1 & w^{-2} & w^{-4} & \ldots & w^{-2\left(N_{c}-1\right)} \\
1 & \vdots & \vdots & & \vdots \\
1 & w^{-\left(N_{c}-1\right)} & w^{-2\left(N_{c}-1\right)} & \ldots & w^{-\left(N_{c}-1\right)^{2}}
\end{array}\right)
$$

where $w=e^{\frac{-j 2 \pi}{N_{c}}}$.

Second, to shelter the signal from the ISI (Inter-Symbol Interference) we add the cyclic prefix (CP) or what are commonly called the guard interval, we can express this step mathematically by multiplying the signal $z$ by :

$$
\boldsymbol{\xi}_{1}=\left(\left(\begin{array}{cc}
0 & \boldsymbol{I}_{N_{g}} \\
& \boldsymbol{I}_{N_{c}}
\end{array}\right) \otimes \boldsymbol{I}_{N_{t}}\right)
$$


where $I$ is i.i.d matrix.

Eventually we transmit a OFDM symbol $\boldsymbol{x}_{N_{c} N_{t}, 1}^{p}$ over a selective correlated Rayleigh fading channel $\boldsymbol{H}$, thus:

$$
\boldsymbol{y}=\boldsymbol{H} \cdot \boldsymbol{\xi}_{1} \cdot{\sqrt{N_{c}}}_{\cdot}\left(\boldsymbol{F}^{-1} \otimes \boldsymbol{I}_{N_{t}}\right) \cdot \boldsymbol{x}_{N_{c} N_{t}, 1}^{p}+\boldsymbol{w}
$$

where $\boldsymbol{w}$ is an $N_{t}\left(N_{c}+N_{g}\right)$ white gaussian noise vector.

This calculation fits either with Joint Coding (JC) or Per Antenna Coding (PAC) technique. In fact, in the (JC) method, the information bit stream is first encoded and then converted into $N_{t}$ parallel sub-streams of which each is modulated and mapped onto corresponding antenna. Fig 1 illustrate the (JC) scheme. However in (PAC) scheme, the incoming bit stream is first transformed to $N_{t}$ parallel sub-streams and then encoding is performed per sub-stream. So, basically, the transmitter consists of $N_{t}$ OFDM transmitters among which the information bits are multiplexed.

At the receiver we consider the system is coherent over a selective correlated Rayleigh fading MIMO channel.

First, the cyclic prefix is removed. This is done by discarding the first $N_{g} N_{r}$ samples of $\boldsymbol{y}$,

$$
\widehat{\boldsymbol{y}}=\frac{1}{N_{c}}\left(\boldsymbol{F} \otimes \boldsymbol{I}_{N_{r}}\right) \boldsymbol{\xi}_{2} \boldsymbol{y}
$$

where $\boldsymbol{\xi}_{2}$ is defined as $\left[\mathbf{0}_{N_{c} N_{g}} \boldsymbol{I}_{N_{g}}\right]$ matrix.

Second, the FFT is performed. Together, give results as

$$
\widehat{\boldsymbol{x}_{N_{c} N_{t}, 1}}=\left\{\left(\boldsymbol{F} \otimes \boldsymbol{I}_{N_{t} N_{r}}\right) \boldsymbol{\xi}_{3}\left(\boldsymbol{F}^{-1} \otimes \boldsymbol{I}_{N_{t} N_{r}}\right)\right\}^{-1} \widehat{\boldsymbol{y}}
$$

where $\boldsymbol{\xi}_{3}$ is commonly called the circulant matrix defined as :

$$
\boldsymbol{\xi}_{3}=\boldsymbol{\xi}_{2} \boldsymbol{H} \boldsymbol{\xi}_{1}
$$

The decision vector for each four symbols is then decoded at time $\left(n_{i}, n_{i+1}\right)$ using a sub-optimum decoder like a Zero Forcing or MMSE decoder. In [9] the optimum decoder for the algebraic space time code was the Shnorr-Echnerr or Sphere-Decoder, but the the ZF or the MMSE still a good candidate for such codes, because they reduce the computational load regarding the Shnorr-Echnerr or the Sphere-Decoder without significant performance loss:

$$
\widehat{\boldsymbol{v}}=\boldsymbol{\phi}_{p}^{-1} \times \widehat{\boldsymbol{x}_{4,1}^{p}}
$$

where

$$
\phi_{\text {Golden }}=\frac{1}{\sqrt{5}}\left(\begin{array}{cccc}
\alpha & \alpha \theta & 0 & 0 \\
0 & 0 & i \bar{\alpha} & i \overline{\alpha \theta} \\
0 & 0 & \alpha & \alpha \theta \\
\bar{\alpha} & 0 & 0 & 0
\end{array}\right) \quad \phi_{\text {Tast }}=\frac{1}{\sqrt{2}}\left(\begin{array}{cccc}
1 & \theta & 0 & 0 \\
0 & 0 & \varphi & -\varphi \theta \\
0 & 0 & \varphi & \varphi \theta \\
1 & -\theta & 0 & 0
\end{array}\right) \quad \phi_{\text {Dast }}^{\boldsymbol{\phi}}=\frac{1}{\sqrt{2}}\left(\begin{array}{cc}
1 & \theta \\
1 & -\theta
\end{array}\right)
$$

A In this case we decode each 2 symbols together, thus we slice the received $\boldsymbol{x}_{4,1}^{\text {Dast }}$ into $\boldsymbol{x}_{2,1}^{\text {Dast }}$

\section{PAPR REDUCTION SCHEME: ASTC SEEN AS PAPR REDUCTION SCHEME}

The main idea in this section is to propose the ASTC codes as an alternative solution for reducing PAPR rather than several reduction schemes Fig 2. As an attractive technique for high data rate wireless communication systems, multiple-input multiple-output orthogonal frequency division multiplexing MIMO-OFDM systems exhibit a large peak-to-average power ratio PAPR due to the superposition of the individual signal components (the carriers). The high PAPR brings on the OFDM signal distortion in the nonlinear region of high power amplifier HPA and the signal distortion induces the degradation of bit error rate BER. In the OFDM systems, several PAPR reduction schemes have been proposed to 


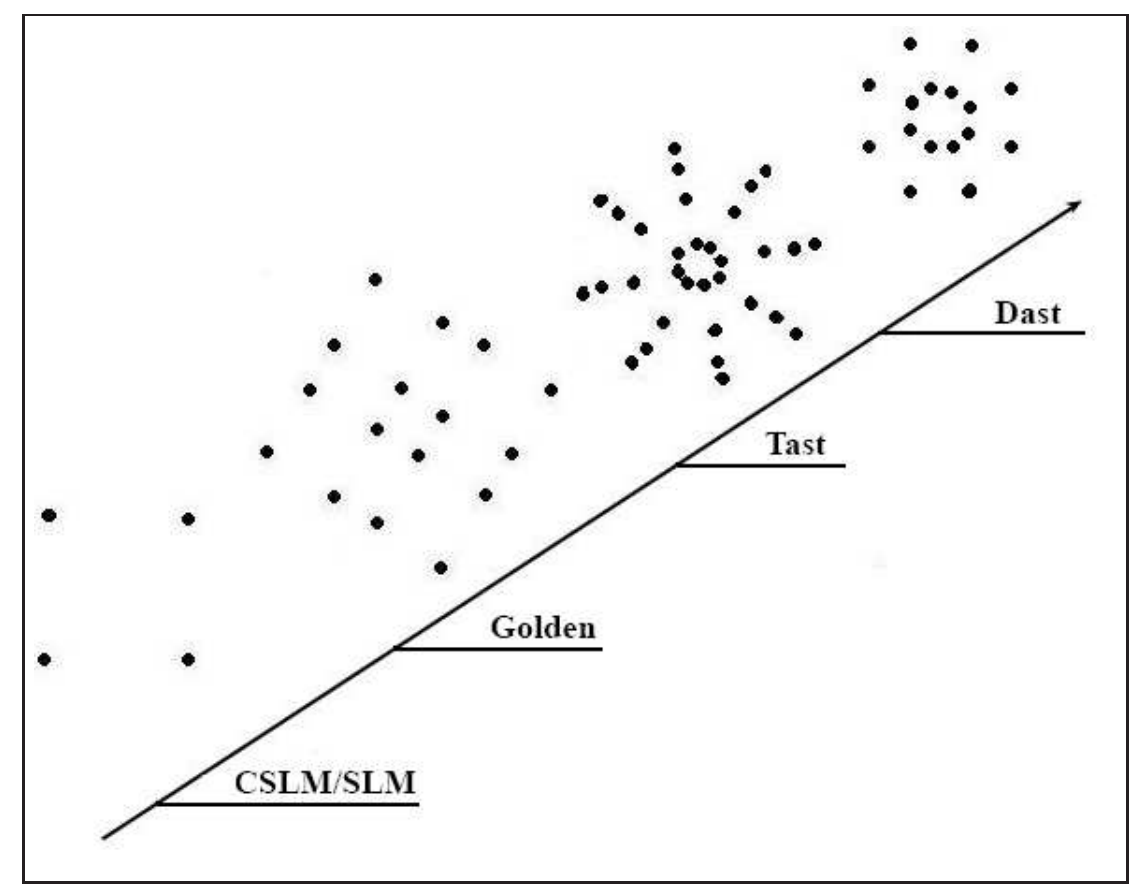

Figure 2. Constellation of Transmitted Signal on PAPR reduction Scheme

solve that problem [11]- [12]. The simple and widely used method is clipping the signal to limit the PAPR below a threshold level, but it causes both in-band distortion and out of band radiation. Another well-known technique to reduce PAPR is block coding, which encode the input data into a codeword with lower PAPR is, but this technique cut down the rate performance. We consider as a benchmark, the selective mapping (SLM) scheme which is an efficient approach for PAPR reduction by generating some statistically independent sequences from same information and transmitting the sequence with the lowest PAPR. Let consider a simple MIMO-OFDM systems with $N_{t}$ transmit antennas that uses $N_{c}$ subcarrier. The resulting baseband OFDM signal $z$ of a block can be expressed as in (8). The PAPR of the transmitted ASTC-MIMO-OFDM signal of (8) is defined as

$$
P A P R=\frac{\max _{\left.1 \leq i \leq\left(N_{t} *\left(N_{c}+N_{g}\right)\right)\right)}|\boldsymbol{z}(i)|^{2}}{E\left(|\boldsymbol{z}(i)|^{2}\right)}
$$

where $E[$.$] denotes the expected value. Then, the complementary cumulative distribution function (CCDF),$ which is the probability that the PAPR of an OFDM symbol exceeds the given threshold $P A P R_{0}$, can be expressed as

$$
C C D F=\operatorname{Pr}\left(P A P R>P A P R_{0}\right)
$$

In the individual SLM (ISLM) [5], $K$ statistically independent sequences are generated for each antenna by multiplying the fixed $N_{c}$-dimensional phase vectors $Q^{K}$ to the input sequences, where $\boldsymbol{Q}^{K}=$ $\left[\boldsymbol{Q}_{0}^{k}, \boldsymbol{Q}_{1}^{k}, \ldots \boldsymbol{Q}_{N_{c}-1}^{k}\right]^{T}, 1 \leq k \leq K$ with each component $\boldsymbol{Q}_{n}^{k} \in\{ \pm 1, \pm j\}$. Consequently, the CCDF of the PAPR of the OFDM signals at each transmit antenna is written as

$$
\operatorname{Pr}\left(P A P R>P A P R_{0}\right)=\left[1-\left(1-\exp \left(-P A P R_{0}\right)\right)^{N_{c}}\right]^{K}
$$

In the Concurrent SLM-based MIMO-OFDM system, the $N_{c}$ subcarrier sequences from $N_{t}$ transmit antennas are multiplied with the same sequence, which is one of $k^{\text {th }}$ sequences from $Q^{K}$. In fact, in the SLM or CSLM, the bit stream at each antenna is scrambled by the best sequence that gives the lowest PAPR, this results in the Symbol Interference (SI) of $\log _{2} K$ bits at each transmit antenna, however the (SI) decreases dramatically the bit error rate performances. By the way we propose to keep the same 
constellation and rather then scrambling the signal by $\{ \pm 1, \pm j\}$ we apply the ASTC encoder. Thus we enjoy triple win: First the BER performance increase significantly, second the rate is enhanced and third the PAPR is reduced thanks to the redundancy of the bit sequence stream transmitted. As a result we consider the ASTC codes like a good compromise between a PAPR reduction scheme and the BER performances.

\section{Empirical Bit Error Rate Calculation : Coherent Frequency Selective Channel CASE}

In order to see in closer window the design criteria of the system design we would like to analyze in this section the pairwise error probability (PEP), we denote by $\boldsymbol{x}_{1}$ and $\boldsymbol{x}_{2}$ tow ASTC-MIMO-OFDM complex Gaussian symbols such as:

$$
\begin{aligned}
& \boldsymbol{x}_{1}=\left[\boldsymbol{x}_{1}(0), \boldsymbol{x}_{1}(1), \boldsymbol{x}_{1}(2), \ldots, \boldsymbol{x}_{1}\left(N_{t}\left(N_{c}+N_{g}\right)\right)\right]^{T} \\
& \boldsymbol{x}_{2}=\left[\boldsymbol{x}_{2}(0), \boldsymbol{x}_{2}(1), \boldsymbol{x}_{2}(2), \ldots, \boldsymbol{x}_{2}\left(N_{t}\left(N_{c}+N_{g}\right)\right)\right]^{T}
\end{aligned}
$$

The probability that, when $\boldsymbol{x}_{1}$ is transmitted, over an ASTC-MIMO-OFDM system, the received point is closer to $\boldsymbol{x}_{2}$ than to $\boldsymbol{x}_{1}$. For a coherent channel $\boldsymbol{H}$, the pairwise error probability is performed as in [13][14]:

$$
\begin{aligned}
P_{e} & =\operatorname{Prob}\left(\boldsymbol{x}_{1} \rightarrow \boldsymbol{x}_{2} \mid \boldsymbol{H}, \boldsymbol{x}_{1} \text { transmit }\right) \\
& =\boldsymbol{Q}\left(\frac{\left\|\boldsymbol{H}\left(\boldsymbol{x}_{1}-\boldsymbol{x}_{2}\right)\right\|^{2}}{2 \sigma_{n}^{2}}\right)
\end{aligned}
$$

We derive first an upper Chernoff bounds for the conditional error probability [15] [16] [14]:

$$
P_{e} \leq \exp \left(\boldsymbol{Q}\left(\frac{\left\|\boldsymbol{H}\left(\boldsymbol{x}_{1}-\boldsymbol{x}_{2}\right)\right\|^{2}}{4 \sigma_{n}^{2}}\right)\right)=\exp \left(\boldsymbol{Q}\left(\frac{\|\boldsymbol{y}\|^{2}}{4 \sigma_{n}^{2}}\right)\right)
$$

We define $\boldsymbol{y}$ as :

$$
\boldsymbol{y}=\boldsymbol{H}\left(\frac{\boldsymbol{x}_{1}}{\sigma_{x_{1}}}-\frac{\boldsymbol{x}_{2}}{\sigma_{x_{2}}}\right)
$$

Since $\boldsymbol{y}$ is a hermitian matrix and if we suppose that $\sigma_{x_{1}}=\sigma_{x_{2}}=\sigma_{s}$ then $\Longrightarrow$ :

$$
\text { (24) } \Rightarrow P e \leq \exp \left(\frac{-\sigma_{s}^{2}}{4 \sigma_{n}^{2}} \boldsymbol{y}^{H} \times \boldsymbol{y}\right)
$$

By averaging over the all frequency selective Channel coefficients $\Rightarrow$ that means we are averaging mathematically over all $\boldsymbol{y}$ then :

$$
P e \leq \int_{y} \exp \left(\frac{-\sigma_{s}^{2}}{4 \sigma_{n}^{2}} \boldsymbol{y}^{H} \times \boldsymbol{y}\right) P(\boldsymbol{y}) d \boldsymbol{y}
$$

If we use (41) the inequality number (27) becomes:

$$
\begin{array}{r}
P e \leq \int_{y} \exp \left(\frac{-\sigma_{s}^{2}}{4 \sigma_{n}^{2}} \boldsymbol{y}^{H} \boldsymbol{y}\right) \frac{1}{\operatorname{det}\left(\pi \boldsymbol{Q}_{y}\right)} \exp \left(-(\boldsymbol{y})^{H} \boldsymbol{Q}_{y}^{-1}(\boldsymbol{y})\right) d \boldsymbol{y} \\
P e \leq \int_{y} \frac{1}{\operatorname{det}\left(\pi \boldsymbol{Q}_{y}\right)} \exp \left(\frac{-\sigma_{s}^{2}}{4 \sigma_{n}^{2}} \boldsymbol{y}^{H} \boldsymbol{y}-(\boldsymbol{y})^{H} \boldsymbol{Q}_{y}^{-1}(\boldsymbol{y})\right) d \boldsymbol{y} \\
P e \leq \int_{y} \frac{1}{\operatorname{det}\left(\pi \boldsymbol{Q}_{y}\right)} \exp \left(-\boldsymbol{y}^{H}\left(\frac{\sigma_{s}^{2}}{4 \sigma_{n}^{2}} \boldsymbol{y}+\boldsymbol{Q}_{y}^{-1}(\boldsymbol{y})\right)\right) d \boldsymbol{y} \\
P e \leq \int_{y} \frac{1}{\operatorname{det}\left(\pi \boldsymbol{Q}_{y}\right)} \exp \left(-\boldsymbol{y}^{H}\left(\frac{\sigma_{s}^{2}}{4 \sigma_{n}^{2}} \boldsymbol{I}_{N t}+\boldsymbol{Q}_{Y}^{-1}\right) \boldsymbol{y}\right) d \boldsymbol{y}
\end{array}
$$


Now if we define $\boldsymbol{B}=\left(\frac{\sigma_{s}^{2}}{4 \sigma_{n}^{2}} \boldsymbol{I}_{N t}+\boldsymbol{Q}_{Y}^{-1}\right)^{-1}$

$$
P e \leq \int_{y} \frac{1}{\pi \operatorname{det}\left(\boldsymbol{Q}_{y}\right)} \exp \left(-\boldsymbol{y}^{H}\left(\boldsymbol{B}^{-1}\right) \boldsymbol{y}\right) d \boldsymbol{y}
$$

And if we multiply $\operatorname{det}(\boldsymbol{B})$ and we divide by $\operatorname{det}(\boldsymbol{B})$ we obtain :

$$
\begin{array}{r}
P e \leq \frac{\operatorname{det}(\boldsymbol{B})}{\operatorname{det}\left(\boldsymbol{Q}_{y}\right)} \int_{Y} \frac{1}{\pi \operatorname{det}(\boldsymbol{B})} \exp \left(-\boldsymbol{y}^{H}\left(\boldsymbol{B}^{-1}\right) \boldsymbol{y}\right) d \boldsymbol{y} \\
P e \leq \frac{\operatorname{det}(\boldsymbol{B})}{\operatorname{det}\left(\boldsymbol{Q}_{y}\right)} \int_{y} \boldsymbol{P}(\boldsymbol{y}) d \boldsymbol{y}
\end{array}
$$

and as

$$
\begin{gathered}
\int_{y} \boldsymbol{P}(\boldsymbol{y}) d \boldsymbol{y}=1 \\
P e \leq \frac{\operatorname{det}(\boldsymbol{B})}{\operatorname{det}\left(\boldsymbol{Q}_{y}\right)}=\frac{\operatorname{det}\left(\frac{\sigma_{s}^{2}}{4 \sigma_{n}^{2}} \boldsymbol{I}_{N_{t}}+\boldsymbol{Q}_{y}^{-1}\right)^{-1}}{\operatorname{det}\left(\boldsymbol{Q}_{y}\right)} \\
P e \leq \operatorname{det}\left(\boldsymbol{I}_{N_{t}}+\frac{\sigma_{s}^{2}}{4 \sigma_{n}^{2}} \boldsymbol{Q}_{y}\right)^{-1}
\end{gathered}
$$

The development given in the appendix below in (40) let us to conclude:

$$
P e \leq\left(1+\frac{\sigma_{s}^{2}}{4 \sigma_{n}^{2}} N_{t}+\sum_{i=1}^{N_{i}} \lambda_{i}+\left(\frac{\sigma_{s}^{2}}{4 \sigma_{n}^{2}}\right)^{N_{i} N_{t}}\left(\prod_{i=1}^{N_{i}} \lambda_{i}\right)^{N_{t}}\right)^{-1}
$$

where $\lambda_{i}$ is the $i^{\text {th }}$ eigenvalues of $\boldsymbol{Q}_{Y}$ matrix.

\section{SIMULATION RESULTS}

To investigate the performance of the proposed space time code, a series of Monte Carlo simulations were carried out. Transmitted symbols belong to 4-QAM. All simulations were performed for at least 100 transmission bit errors, and BER curves were averaged over 50 Monte Carlo trials. Figures below show the comparative simulation results.

\section{A. ASTC transmission improvement}

We have assumed that the normalized Doppler frequency is perfectly known and remains constant. We apply the correlated channel model described in II-E.

1) Time variation channel case: This subsection compares the BER performance of the ASTC and the classical transmitter. The time and the guard spacing are respectively $N_{t}=2$ and $N_{g}=32$. The number of used subcarriers is $N_{c}=128$. Fig 4 depicts the system performances in a time variation channel conditions. The performances of the system without ASTC codes is also included to serve as a benchmark. We notice that the ASTC-MIMO-OFDM transmitter outperforms the classical one. When the SNR is up to $20 \mathrm{~dB}$ the gain obtained is significant.The Golden code overcame the DAST and TAST codes. In fact the gain obtained by the Golden code is about $1 \mathrm{~dB}$ for a BER equal to $10^{-4}$ regarding the TAST code. However, the improvement is negligible with the DAST codes, but we are coding 4 symbols with the Golden codes 
rather than 2 symbols with the DAST code, so the gain in terms of rate is important. The Golden codes rate is two times the DAST's rate.

2) Static channel case: Fig 3 shows the BER performance when the time variation is not satisfied. The diamond curve corresponds to the performance of a classical Transmitter. The circled curve gives the best performance corresponding to the Golden codes. It can be seen that performance degradation is more significant in the case of the classical receiver. In fact, the ASTC, alleviates the degradation mainly when the SNR is higher than $15 \mathrm{~dB}$.

\section{B. Doppler frequency effect}

Fig 6, depicts the system performances for three normalized doppler frequencies, when the vehicle speed $\boldsymbol{v}_{h}=30 \mathrm{~km} / \mathrm{h}$ for systems operating in the $9 \mathrm{GHz}$ band, $f_{d}=f_{m} T=0.01, f_{d}=0.05$ and $f_{d}=0.005$. In this figure the Golden codes are used as an ASTC code. When $f_{d}=0.005$, the gain obtained is about $2 \mathrm{~dB}$ and $4 \mathrm{~dB}$ for a BER of $10^{-2}$ respectively between $f_{d}=0.05$ and $f_{d}=0.01$. It is noted that performances are better when the normalized fading rate $f_{d}$ decreases.

\section{Pairwise Error Probability}

The PER performance is used to better understand the system design criteria. The main goal of this subsection is to compare the the proposed ASTC codes to each other in terms of PEP and to compare the similarity of the PEP to BER performance simulated on Fig 3. When comparing the Fig 3 through Fig 5, the slope of the both figures remain the same this is thanks to the diversity order of 4 with a small differences mainly when we compare the TAST and Golden BER versus the PEP's one caused by the unused subcarriers $N_{g}$. We can see for small SNR the term consisting of the summation of the eigenvalues in (5) will dominate the bounds. For asymptotically high SNRs, however, the term containing the products of the eigenvalues will be dominant, resulting in the diversity and coding gain criteria.

\section{PAPR reduction scheme}

1) ISLM versus ASTC: In Fig 7, 8, 9 the CCDF is plotted for the ISLM (Individual Selected Mapping). For reference, the CCDF of original OFDM signals with PAPR reduction (ISLM) without ASTC codes is included. It is clearly that ISLM combined with ASTC show much better performance than conventional ISLM. In fact, Fig 7 shows, Golden+ISLM for a saturation probability levels in the order of $10^{-2}$, the gain is around $1 \mathrm{~dB}$ regarding the ISLM and $0.5 \mathrm{~dB}$ versus Golden scheme. Fig 8 shows, for a saturation probability levels in the order of $10^{-3}$, the gain remains around $1 \mathrm{~dB}$ compared to the simple ISLM mode. Furthermore if we compare the CCDF of original MIMO-OFDM signals with ISLM reduction versus the ASTC-MIMO-OFDM in Figs 7, 8, 9, the gain still remain noticeable for saturation probability levels up to the order of $10^{-2}$, either for Golden or Tast codes.

2) CSLM versus ASTC : In Fig 11, 10, 12 the CCDF is plotted for the CSLM (Concurrent Selected Mapping). For reference, the CCDF of original OFDM signals with PAPR reduction (CSLM) without ASTC codes. It is clearly that CSLM combined with ASTC shows much better performance than conventional CSLM. In fact, Fig 11 shows, Golden+CSLM for a saturation probability levels in the order of $10^{-3}$, the gain is around $3 \mathrm{~dB}$ regarding the CSLM and Golden scheme. Fig 10 shows, for a saturation probability levels in the order of $10^{-3}$, the gain remains around $3 \mathrm{~dB}$ compared to the simple CSLM mode and about $1.8 \mathrm{~dB}$ for the Tast mode. Furthermore if we compare the CCDF of original MIMO-OFDM signals with CSLM reduction versus the ASTC-MIMO-OFDM in Figs 11 and 12 , the gain still notable for saturation probability levels up to the order of $10^{-3}$, for Tast codes the gain is negligible. 


\section{CONCLUSION}

It has been shown that by their construction, the Algebraic Space Time Codes have a reasonable BER when working in a non selective channel [8] thanks to their properties, full rank, full rate, and nonvanishing determinant for increasing rate. In this paper, we have proved that it is possible to combine MIMO-OFDM system with ASTC encoder under a frequency-selective channel. Computer simulations have demonstrated the efficiency of the proposed ASTC encoder either in terms of performance improvement or in terms of PAPR reduction. Another interesting research area consists of applying the ASTCMIMO-OFDM discussed in this paper to the Ultra Wide Band (UWB) systems under a non coherent channel conditions.

\section{APPENDIX A}

\section{MATHEMATIC TOOLS USED FOR THE EMPIRICAL BER}

$\boldsymbol{A}$ is a hermitian matrix $\Rightarrow \boldsymbol{A}=\boldsymbol{A}^{H}$. $\|\boldsymbol{A}\|_{2}^{2}=\boldsymbol{A} \times \boldsymbol{A}$.

$$
\operatorname{det}\left(\boldsymbol{I}_{N t}+\alpha \boldsymbol{A}\right)=1+\alpha \sum_{n=1}^{N} a_{x x}+\alpha^{2} \sum_{n=1}^{N-1} \sum_{y=x+1}^{N} \operatorname{det}\left(\begin{array}{cc}
a_{x x} & a_{x y} \\
a_{y x} & a_{y y}
\end{array}\right)+\ldots
$$

$Q_{\boldsymbol{y}}=E\left(\boldsymbol{y} \boldsymbol{y}^{H}\right)=$ the Covariance matrix of $\boldsymbol{y}$

For a hermitian matrix $\boldsymbol{Z}$ of covariance $\boldsymbol{Q}_{z}$ with $E(\boldsymbol{Z})=\mu_{z}$ mean, the probability density of $\boldsymbol{Z}$ is performed as [1]:

$$
P(\boldsymbol{Z})=\operatorname{det}\left(\pi \boldsymbol{Q}_{z}\right)^{-1} \exp \left(-\left(\boldsymbol{Z}-\mu_{z}\right)^{H} \boldsymbol{Q}_{z}^{-1}\left(\boldsymbol{Z}-\mu_{z}\right)\right)
$$

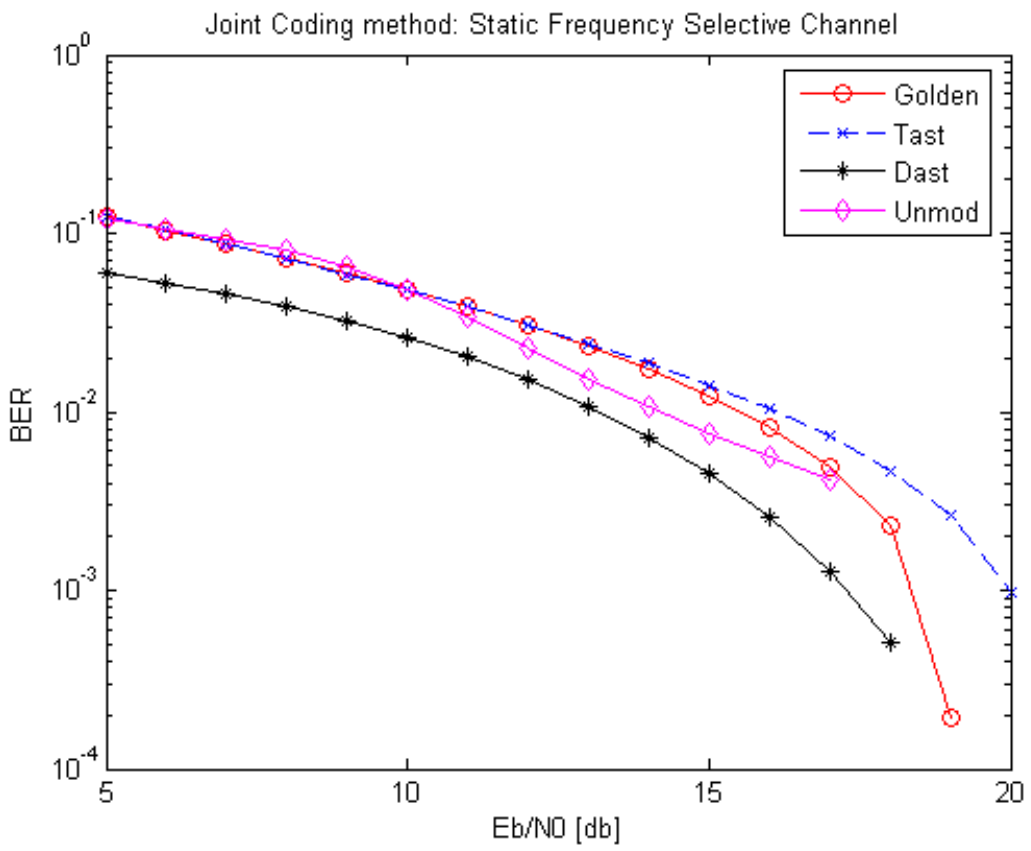

Figure 3. Joint Coding method: Static Frequency Selective Channel 


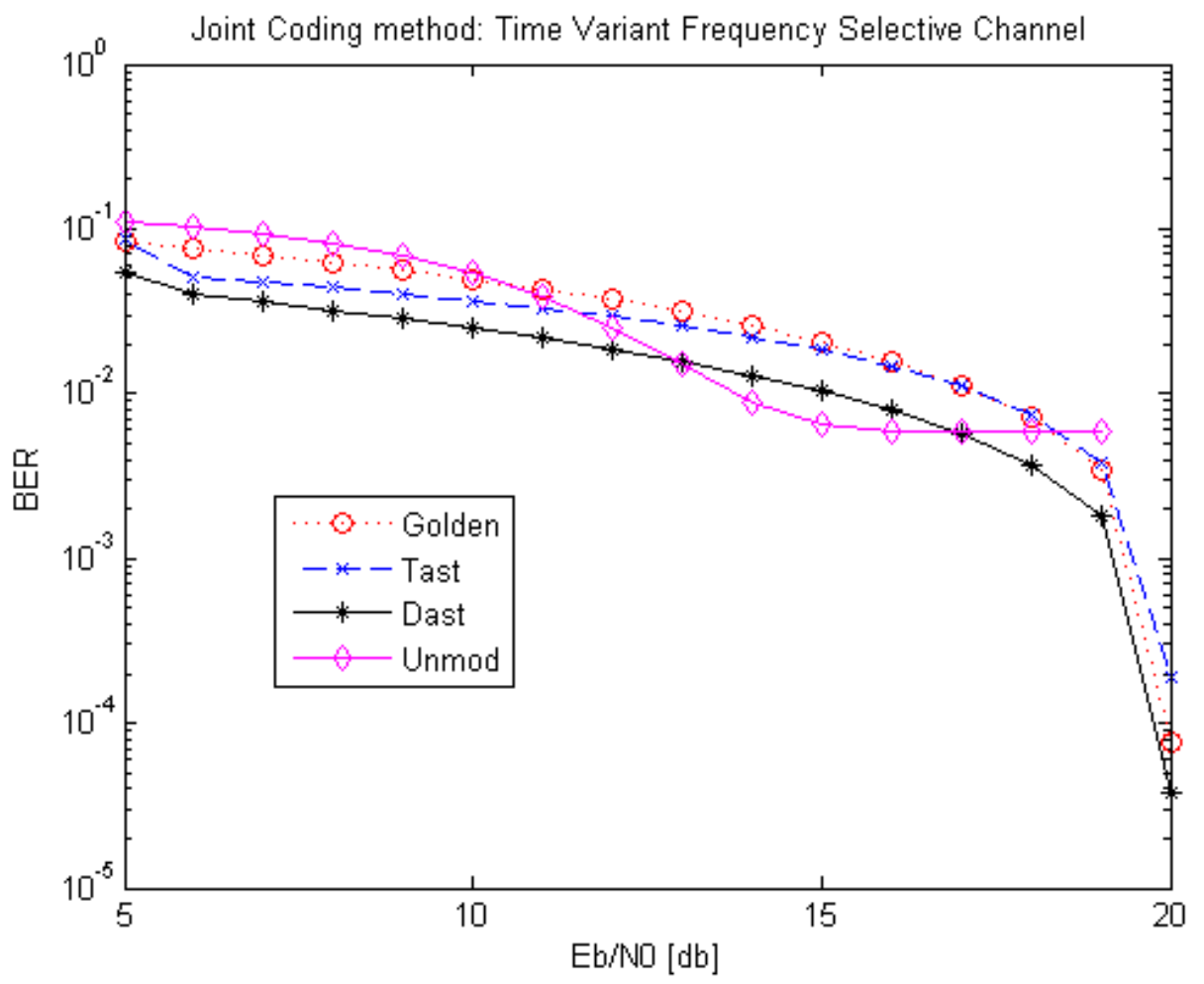

Figure 4. Joint Coding method: Time Variant Frequency Selective Channel

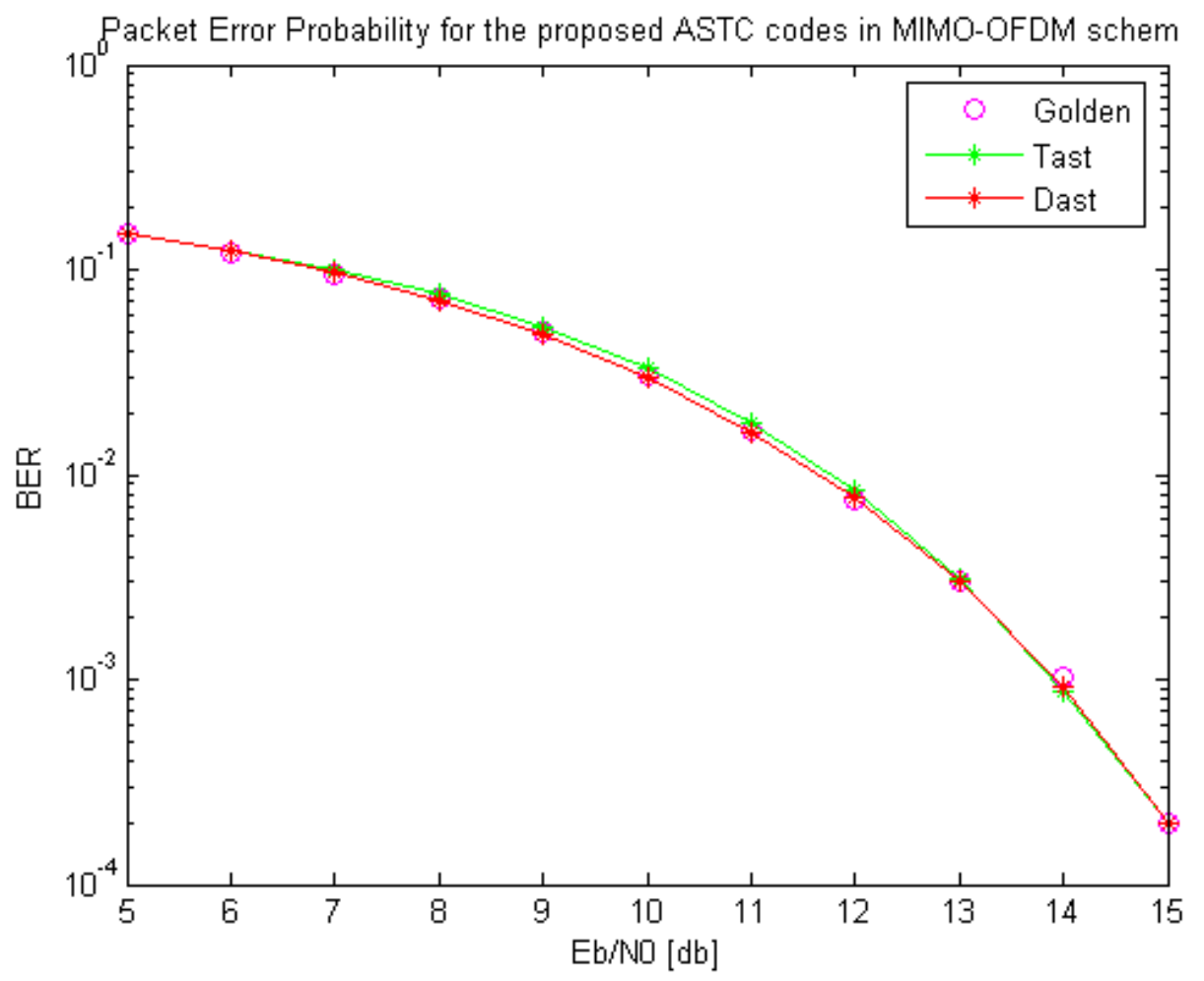

Figure 5. Pairwise Error Probability 


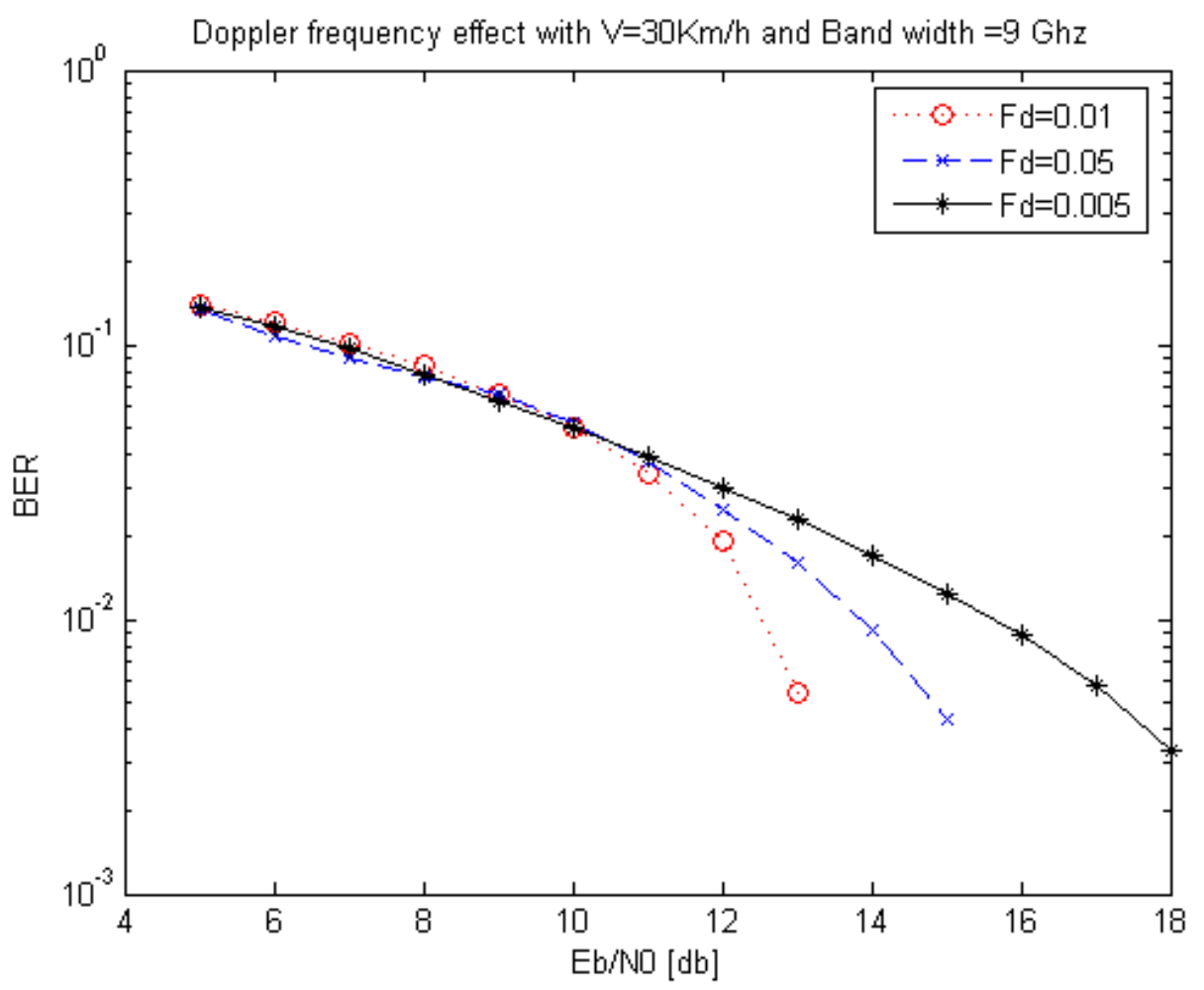

Figure 6. Normalized doppler frequency effect with $\mathrm{V}=30 \mathrm{Km} / \mathrm{h}$ and Band width $=9 \mathrm{Ghz}$

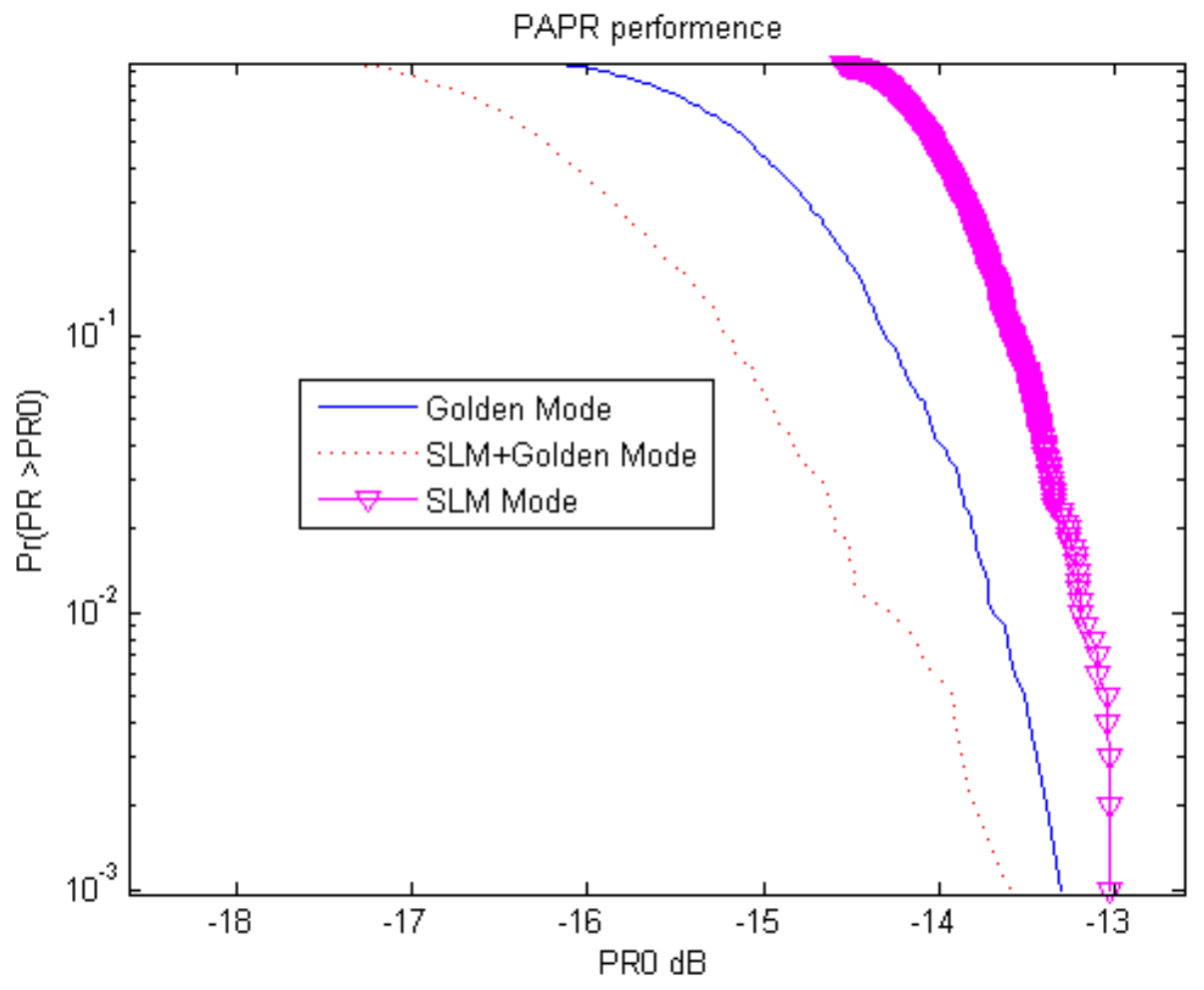

Figure 7. PAPR reduction scheme for SLM and Golden 


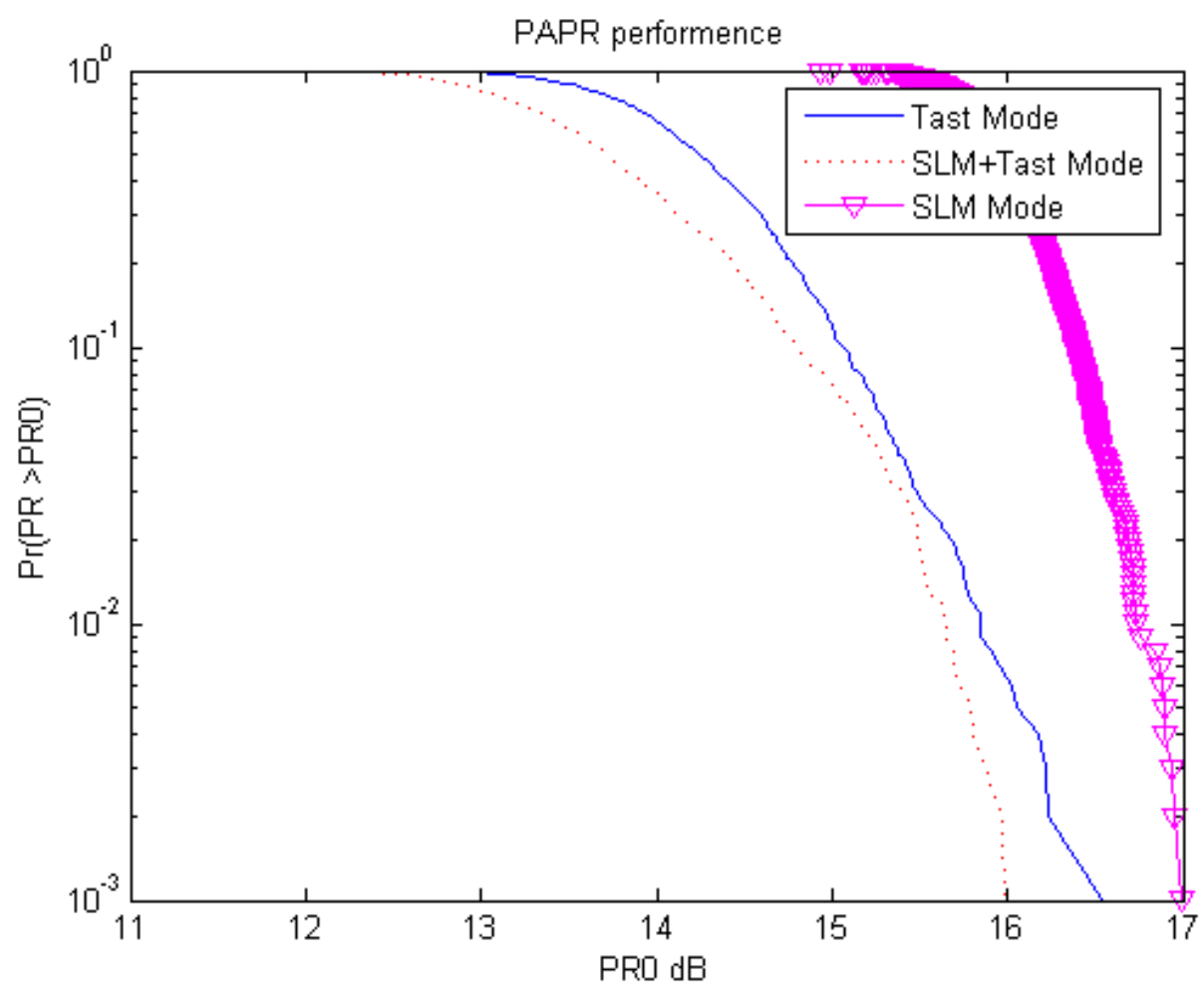

Figure 8. PAPR reduction scheme for SLM and Tast

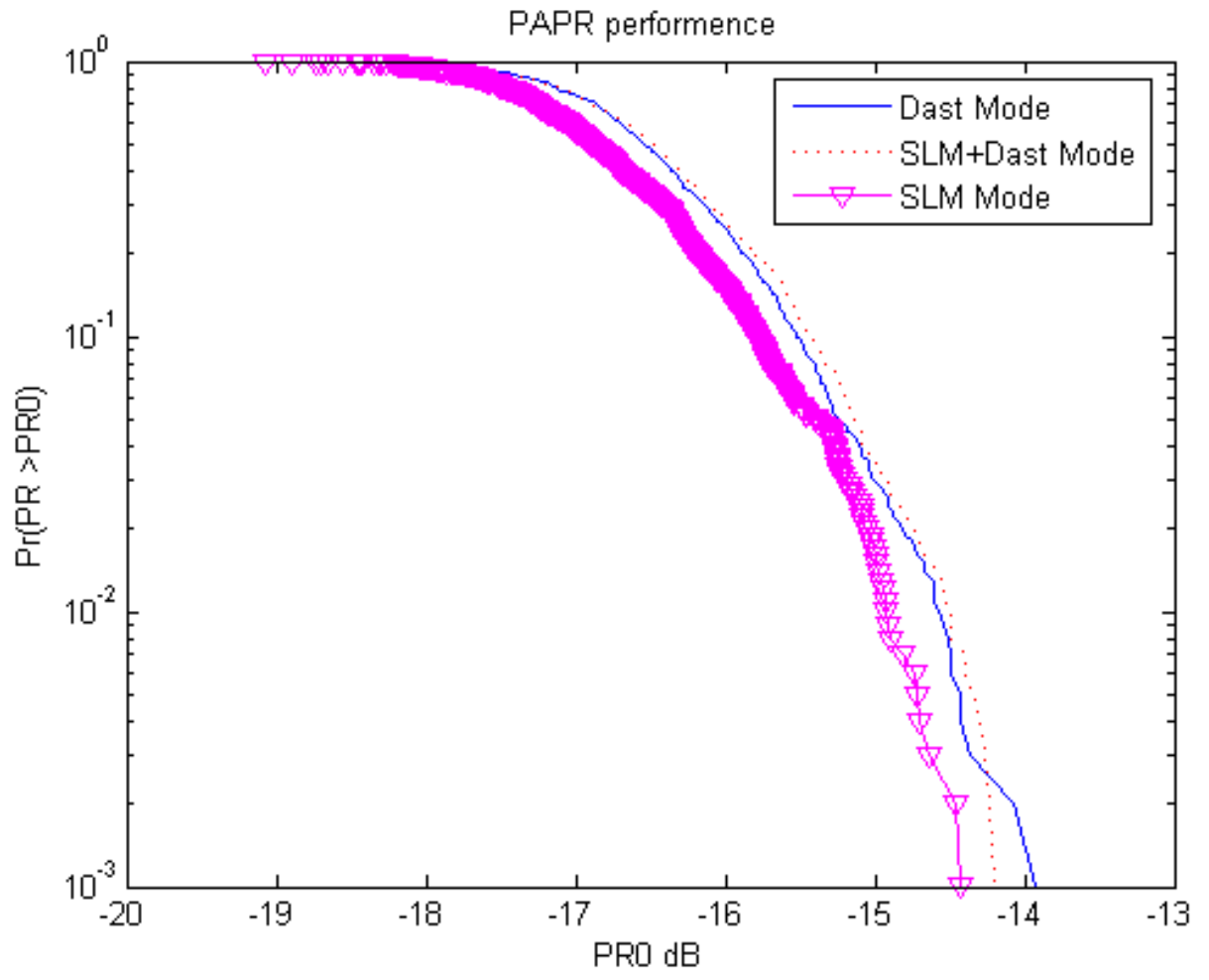

Figure 9. PAPR reduction scheme for SLM and Dast 


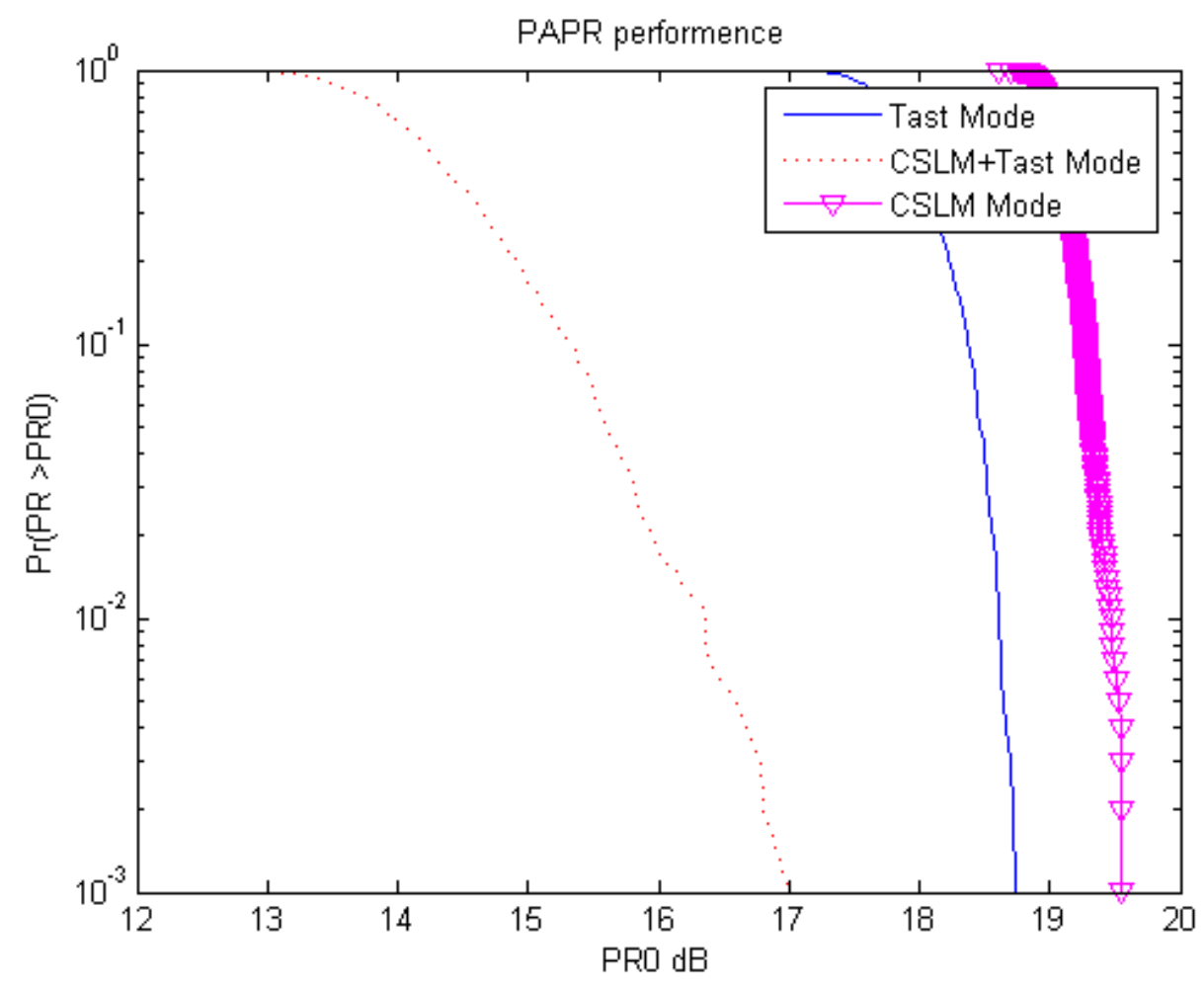

Figure 10. PAPR reduction scheme for CSLM and TAST

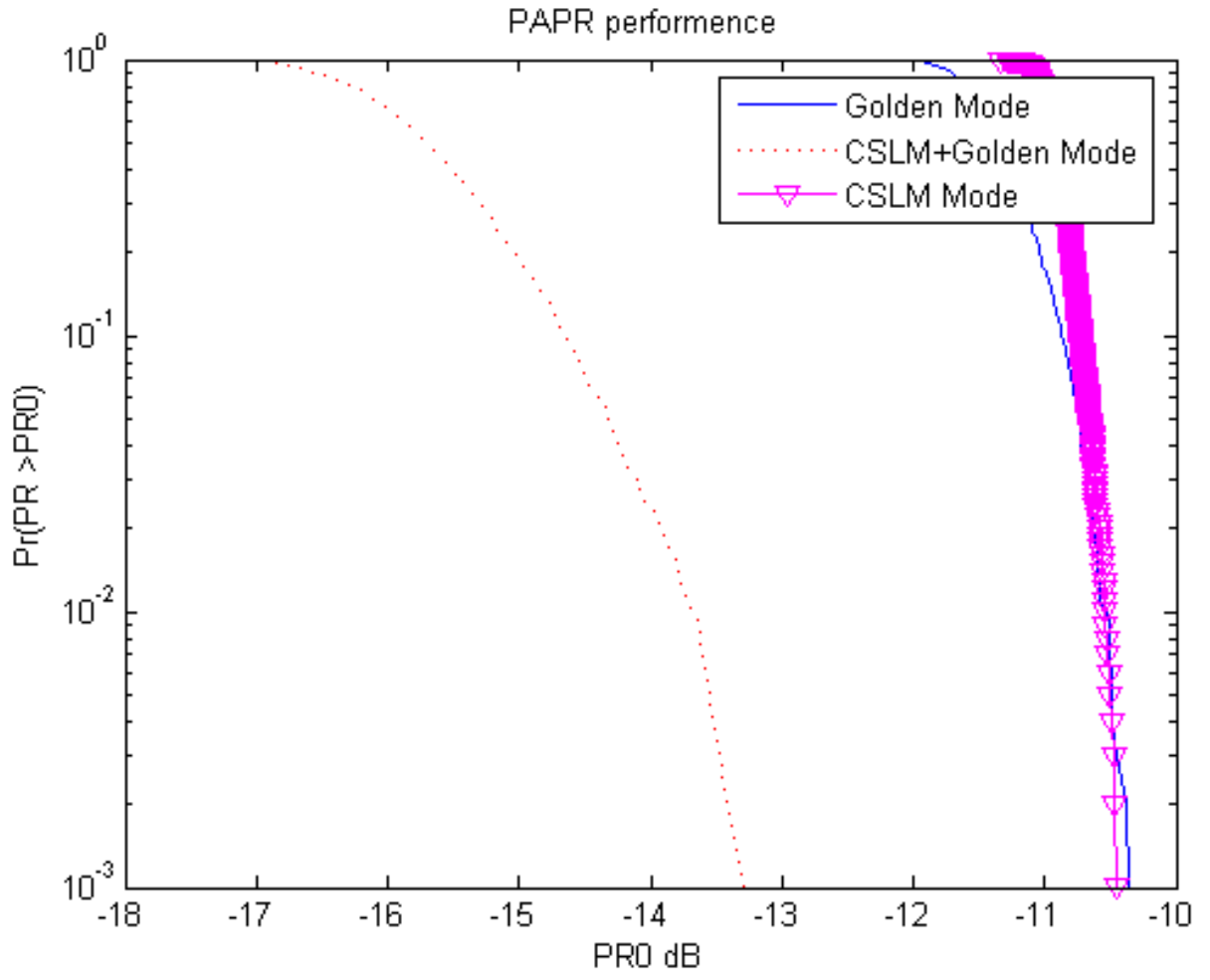

Figure 11. PAPR reduction scheme for CSLM and Golden 


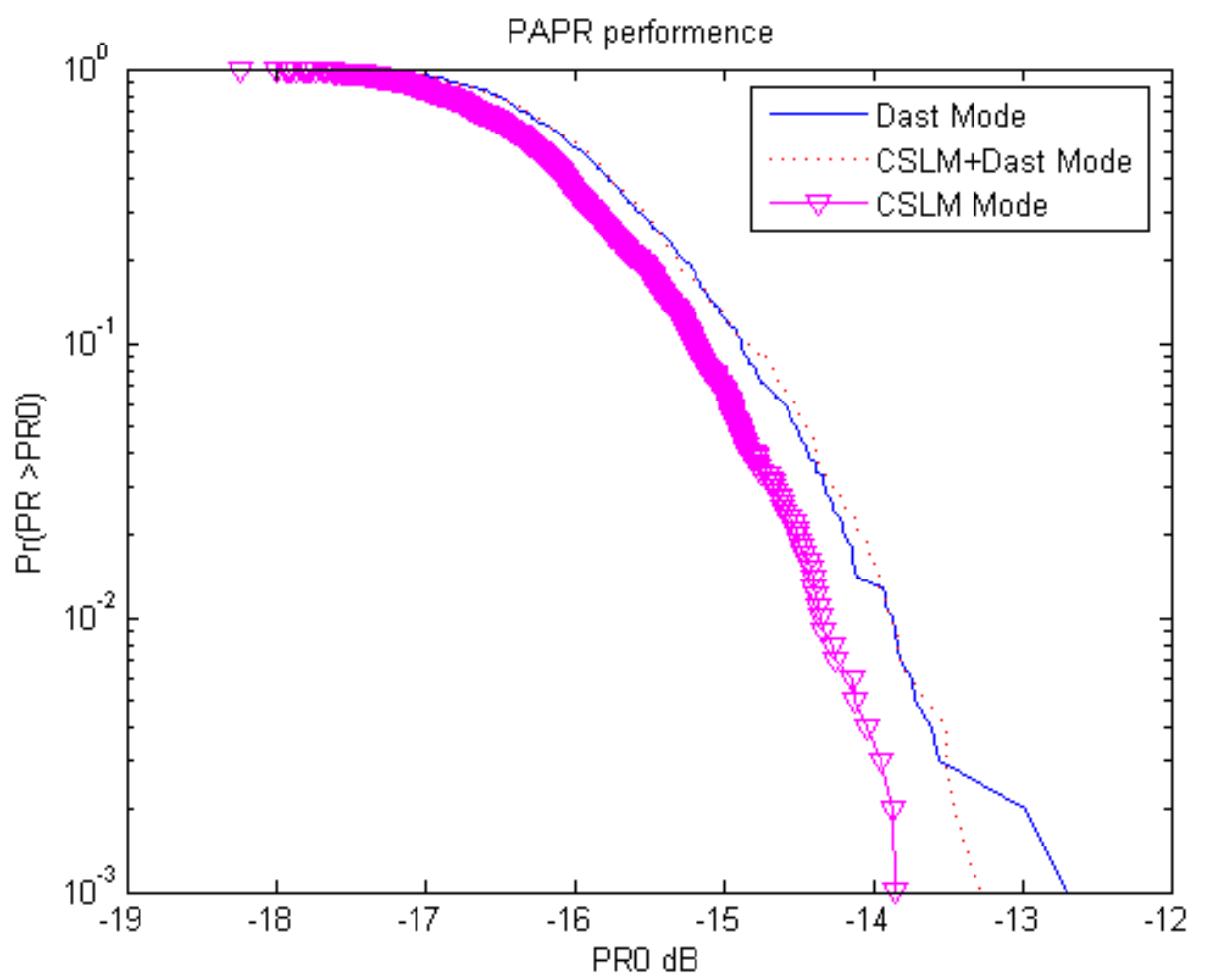

Figure 12. PAPR reduction scheme for CSLM and Dast

\section{REFERENCES}

[1] J.Bingam, "Multicarrier modulation for data transmission: An idea whose time has come," IEEE Commun. Mag., vol. 28, pp. 5-14, Jan. 1990.

[2] S. Weinstein and P. Ebert, "Data transmission by frequency-division multiplexing using the discrete fourier transform,", IEEE Trans. Wireless Commun., vol. 19, pp. 628-634, 10 octobre 1971.

[3] H. Sari and I. Jeanclaude, "An analysis of orthogonal frequency-division multiplexing for mobile radio applications," Proc., IEEE Veh. Technol. Conf., 1994.

[4] S. H. Muller and Al, OFDM With Reduced Peak-to-Average Power Ratio by Multiple Signal Representation. Annals of Telecommunications, February 1997, vol. 50, no. 1-2.

[5] J. Gao, J. Wang, and Z. Xie, "Peak to average power ratio reduction for MIMO-OFDMsystems with decomposed selected mapping," IJISS, vol. 3, pp. 572-580, 2009.

[6] H. Bolcskei and E. Zurich, "MIMO-OFDM wireless systems: basics, perspectives, and challenges," IEEE Trans. Wireless Commun., vol. 13, pp. 31-37, Aug. 2006.

[7] J. C. Belfiore, G. Rekaya, and E. Viterbo, "The golden code: a 2 x 2 full-rate space-time code with non-vanishing determinants," IEEE Trans. Inform. Theory, vol. 13, pp. 67-75, 2004.

[8] A. Bannour, M. L. Ammari, and R. Bouallegue, "Analysis of ASTC in a correlated rayleigh fading channel with imperfect channel estimation," International Conference on Advanced Communication Technology, 2010.

[9] G. R. ben Othman, "Nouvelles constructions algébriques de codes spatio-temporels atteignant le compromis Ťmultiplexage-diversitéŤ," Ph.D. dissertation, ENST, 2004.

[10] M. L. Ammari and F. Gagnon, "Iterative channel estimation and decoding of turbo-coded OFDM symbols in selective rayleigh channel," Canadian J. Elect. Comput. Eng., vol. 32, no. 1, pp. 9-18, 2007.

[11] R. F. Fischer and M. Hoch, "Peak-to-average power ratio reduction in mimo ofdm."

[12] R. F and H. Fischer, "Peak-to-average power ratio PAR reduction in ofdm based on lattice decoding."

[13] F. Oggier, "Algebraic methods for chanel coding," Ph.D. dissertation, EPFL, 2005.

[14] V. tarohk N. Seshadri and A. R. Calderbank, "Space-time block codes for hight data rate wireless communications: performence criterion and code constration," IEEE Trans. Inform. Theory, vol. 45, pp. 1456-1467, 1999.

[15] H. Bolcskeis and A. J. Paulraj, "Space-frequency coded braod-band systems," Proc., IEEE Wireless Communications and Networking Conference, vol. 1-2000, pp. 1-6, 2000.

[16] J. G. Proakis, Digital Communication. McGraw-Hill Series in Electrical and computer Engineering. 


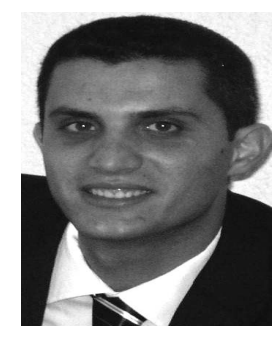

Ahmed BANNOUR was born in Moknine, Tunisia,in 1982. He received the engineering degree and the M.Sc respectively in Telecommunications in 2006 and in Communications System in 2008 from the National Engineering School of Tunis, Tunisia. From 2006 to 2009, he was a Research Associate with the Laboratory of Communications System, National Engineering School of Tunis, Tunisia. He is currently an Assistant at the High Institute of Computer Science Mahdia. His research interests include MIMO, OFDM systems, Space Time Code.

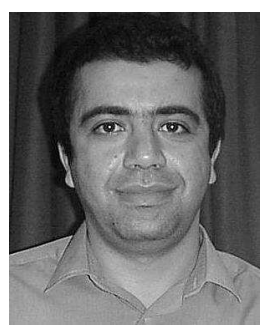

Mohamed Lassaad Ammari was born in Mahdia, Tunisia, in 1972. He received the engineering degree from the t' Ecole Supt'erieure des Communications, Tunis, Tunisia, in 1995, and the M.Sc. and Ph.D. degrees from Universitt'e Laval, Quebec, Quebec, Canada, in 2000 and 2003, respectively. From 2003 to 2005, he was a Research Associate with the Laboratory of Communications and Integrated Microelectronics (LACIME), t' Ecole de Technologie Supt'erieure, Montreal, Quebec, Canada. He is currently an Assistant Professor at the t' Ecole Nationale dŠIngt'enieurs de Sousse. His research interests include channel equalization, OFDM systems, turbo detection, and adaptive modulation.

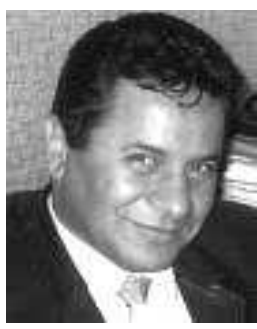

Ridha BOUALLEGUE is Professor at the National Engineering School of Tunis, Tunisia (ENIT), he practices at the Superior School of Communications of Tunis (Sup'Com). He is founding in 2005, and Director of the Research Unit "Telecommunications Systems : 6'Tel@Sup'ComŤ. He is founding in 2005, and Director of the National Engineering School of Sousse. He received his PhD in 1998 then HDR in 2003 on multiuser detection in cellular radio systems of the next generation. His research and fundamental development, focus on the physical layer of telecommunication systems in particular on digital communications systems, MIMO, OFDM, CDMA, UWB, WiMAX, LTE, SDR, ... He has published 2 book chapters, 75 articles in refereed conference lectures and 15 journal articles (2009). 